\title{
Vol. 4, No. 1, 2019 \\ FINDING THE GLOBAL MINIMUM OF THE GENERAL QUADRATIC PROBLEMS DURING DETERMINISTIC GLOBAL OPTIMIZATION IN CYBER-PHYSICAL SYSTEMS
}

\author{
Anatolii Kosolap \\ University of Chemical Engineering, 8, Gagarina av., Dnipro, 49005, Ukraine \\ e-mail:anivkos@ua.fm
}

Submitted on 15.05.2019

(C) Kosolap A., 2019

\begin{abstract}
Cyber-Physical Systems (CPS) are integrations of computation and physical processes. We consider effective computations for designing difficult systems. In this paper, we propose new method of exact quadratic regularization for deterministic global optimization (EQR). This method can be used for the solution of a wide class of multiextreme problems, in particular, general quadratic problems. These problems will be transformed to maximization of norm a vector on convex set. The convex set is approximated by a polyhedron or intersection of balls. We offer the modified dual problem for maximization of norm a vector on intersection of balls. It allows to receive the solution of a primal problem. We use only local search (primal-dual interior point method) and a dichotomy method for search of a global extremum in the general quadratic problems.
\end{abstract}

Index Terms: global optimization, exact quadratic regularization, general quadratic problems, intersection of balls, modified dual theory, test problems.

\section{INTRODUCTION}

Many problems referring to economy, cyber-physical systems, finance, project optimization, planning, computer graphics, management, scheduling, sensor networks and other difficult systems can be transformed to general quadratic optimization problems in finite dimensional space. Computer networking, medical devices, mobile devices, robots, transportation and energy systems can benefit from CPS co-design and optimization techniques. The design, construction and verification of cyber-physical systems pose a multitude of computation challenges that must be addressed by a community of researchers.

Such problems contain many of local minima and belong to NP-difficult class. It is necessary to develop new methods of global optimization for the solution of these problems.

The existing methods in global optimization can be classified as deterministic and probabilistic. Deterministic ones include: Lipschitzian, Branch and Bound, Cutting Plane, Difference of Convex Function, Outer Approximation, Reformulation-Linearization, Interval methods [1]. They demand the exponential number of iterations for finding global extremum. The probabilistic methods include random search, genetic and evolutionary methods [2]. However, these methods allow to find global extremum only with some probability.

Often for the solution of the general quadratic problems we use semidefinite optimization. Generally, semidefinite optimization allows to find only estimations of a global extremum [3].

In this paper, we propose new method of exact quadratic regularization for deterministic global optimization. This method can be used for the solution of a wide class of multiextreme problems, in particular, general quadratic and polynomial problems. The method $\mathrm{EQR}$ includes local methods of optimization and of a dichotomy method. Effective primal-dual interior point methods are used for the solution to this problems [4].

\section{METHOD OF EXACT QUADRATIC REGULARIZATION}

Consider new method of exact quadratic regularization for the solution of the problem of global optimization. We will consider nonlinear programming problems of the form

$$
\min \left\{f_{0}(x) \mid f_{i}(x) \leq 0, i=1, \ldots, m, x \in E^{n}\right\},
$$

where all functions $f_{i}(x)$ are twice differentiable, $x$ is a vector in $n$-dimensional Euclidean space $E^{n}$. Let the solution of a problem (1) exist, its feasible domain is bounded and $x^{*}$ is the point of global minimum (1). We transform the problem (1) to the following one

$$
\min \left\{x_{n+1} \mid f_{0}(x)+s \leq x_{n+1}, f_{i}(x) \leq 0, i=1, \ldots, m, x \in E^{n}\right\},
$$

where the value $s$ is chosen so that $f_{0}\left(x^{*}\right)+s \geq\left\|x^{*}\right\|^{2}$. The solution to the problem (2) is the point $\left(x^{*}, x_{n+1}^{*}\right)$, where $x_{n+1}^{*}=f_{0}\left(x^{*}\right)+s \geq 0$. Further, using the replacement $x=A z$ where matrix $A$ of the order $(n+1) \times(n+1)$ is given by

$$
\left(\begin{array}{cccc}
1 & 0 & \ldots & 0 \\
0 & 1 & \ldots & 0 \\
\ldots & \ldots & \ldots & \ldots \\
z_{1} & z_{2} & \ldots & z_{n+1}
\end{array}\right)
$$


The problem (2) is transformed to the following one

$$
\begin{aligned}
& \min \left\{\|z\|^{2} \mid f_{0}(x)+s \leq\|z\|^{2},\right. \\
& \left.f_{i}(x) \leq 0, i=1, \ldots, m, z \in E^{n+1}\right\},
\end{aligned}
$$

where $z=\left(x, x_{n+1}\right)$. Thus, the problem (1) transformed to the minimization of a norm of square vector. The value $r>0$ exists so that all functions $f_{i}(z)+r\|z\|^{2}$ are convex on the bounded feasible domain of the problem (1). It follows from the fact that Hessians of these functions are positively defined matrixes (matrixes with a dominant main diagonal).

Let us use the quadratic regularization to transform the problem (3) into the following convex problem

$$
\min \left\{d \mid g_{i}(z) \leq d, i=0, \ldots, m, r\|z\|^{2} \leq d\right\}
$$

or

$$
\max \left\{\|z\|^{2} \mid g_{i}(z) \leq d, i=0, \ldots, m, r\|z\|^{2}=d\right\} .
$$

where all $g_{i}(z), i=0, \ldots, m$ are strong convex functions $g_{0}(z)=f_{0}(x)+s+(r-1)\|z\|^{2}, g_{i}(z)=f_{i}(x)+r\|z\|^{2}, i=1$,

Example 1. Then problem

$$
\begin{aligned}
& \min \left\{\|x\|^{2} \mid-4 x_{1}^{2}-x_{2}^{2}-x_{1} x_{2}-2 x_{1}-22 x_{2}+54 \leq 0,\right. \\
& \left.3 x_{1}^{2}-2 x_{2}^{2}+8 x_{1} x_{2}+64 x_{1}-30 x_{2}+102 \leq 0\right\}
\end{aligned}
$$

has 3 local minimums but the problem (4)

$$
\begin{aligned}
& \min \left\{d \mid-4 x_{1}^{2}-x_{2}^{2}-x_{1} x_{2}-2 x_{1}-22 x_{2}+54+2\left(x_{1}^{2}+\right.\right. \\
& \left.+x_{2}^{2}+x_{3}^{2}\right)+54 \leq d, \\
& \left.3 x_{1}^{2}-2 x_{2}^{2}+8 x_{1} x_{2}+64 x_{1}-30 x_{2} 2\left(x_{1}^{2}+x_{2}^{2}+x_{3}^{2}\right)+102 \leq d\right\}
\end{aligned}
$$$$
\text { has only one local minimum. }
$$

Example 2. Then problem

$$
\begin{aligned}
& \min \left\{\left|4-\left(x_{1}-1\right)^{2}-x_{2}^{2}\right|-x_{1}+8 x_{2} \leq 11,\right. \\
& \left.x_{1}+4 x_{2} \leq 7,6 x_{1}+4 x_{2} \leq 17, x_{1} \leq 2.5, x_{2} \leq 2\right\}
\end{aligned}
$$

will be transformed to (5)

$$
\begin{aligned}
& \max \left\{\|z\|^{2} \mid 4-\left(x_{1}-1\right)^{2}-x_{2}^{2}+s+(r-1)\|z\|^{2} \leq d,\right. \\
& \left.-x_{1}+8 x_{2} \leq 11, x_{1}+4 x_{2} \leq 7,6 x_{1}+4 x_{2} \leq 17, x_{1} \leq 2.5, x_{2} \leq 2\right\}
\end{aligned}
$$

where $s=10, r=3$ and $d=33$.4. This problem will have only one local minimum.

Example 3. Then problem

$$
\begin{aligned}
& \min \left\{\|x\|^{2} \mid-4 x_{1}^{2}+5 x_{1}+2 x_{2}^{2}-6 x_{2} \leq\right. \\
& \left.\leq-10,-2 x_{1}^{2}-8 x_{1}-4 x_{2}^{2}+6 x_{2} \leq-5\right\}
\end{aligned}
$$

has 3 local minimums and the problem

$$
\begin{aligned}
& \max \left\{\|x\|^{2}\|x\|^{2}+s+4\|x\|^{2} \leq d,\right. \\
& -4 x_{1}^{2}+5 x_{1}+2 x_{2}^{2}-6 x_{2}+10+5\|x\|^{2} \leq d, \\
& \left.-2 x_{1}^{2}-8 x_{1}-4 x_{2}^{2}+6 x_{2}+5+5\|x\|^{2} \leq d\right\}
\end{aligned}
$$

also has 3 local minimums. After linear transformation of space

$$
\begin{gathered}
\max \left\{\|z\|^{2}\|\mid z-3\|^{2}+s+4\|z\|^{2} \leq d,\right. \\
-4\left(z_{1}-3\right)^{2}+5\left(z_{1}-3\right)+2\left(z_{2}-3\right)^{2}- \\
-6\left(z_{2}-3\right)+10+5\|z\|^{2} \leq d,
\end{gathered}
$$

$$
\begin{aligned}
& -2\left(z_{1}-3\right)^{2}-8\left(z_{1}-3\right)-4\left(z_{2}-3\right)^{2}+ \\
& \left.+6\left(z_{2}-3\right)+5+5\|x\|^{2} \leq d\right\},
\end{aligned}
$$

the problem becomes one-extreme $(s=18)$.

Theorem 1. Let $\left(z^{0}, d_{0}\right)$ be the solution to the problem (5) and the condition (3) holds for the parameter $s$. Then $x^{*}=\bar{z}^{0}$ is the solution to the problem (1).

Proof. We obtain

$$
\begin{aligned}
& f_{0}\left(\bar{z}^{0}\right)+s+(r-1)\left\|z^{0}\right\|^{2} \leq d_{0}, \\
& f_{i}\left(\bar{z}^{0}\right)+r\left\|z^{0}\right\|^{2} \leq d_{0}, \quad i=1, \ldots, m .
\end{aligned}
$$

Taking into account $r\left\|z^{0}\right\|^{2}=d_{0}$, we get

$$
f_{0}\left(\bar{z}^{0}\right)+s \leq\left\|z^{0}\right\|^{2}, \quad f_{i}\left(\bar{z}^{0}\right) \leq 0, \quad i=1, \ldots, m .
$$

The first constraint is equivalent to

$$
f_{0}\left(\bar{z}^{0}\right)+s \leq\left\|\bar{z}^{0}\right\|^{2} \quad\left(z_{n+1}^{0}=0\right) \text { or } f_{0}\left(\bar{z}^{0}\right)+s=\left\|z^{0}\right\|^{2} .
$$

Let $z^{*}$ be the solution to the problem (1) and $m^{z^{*}}=f_{0}\left(\bar{z}^{*}\right)+s, \quad d^{*}=r\left\|z^{*}\right\|^{2}\left(f_{0}\left(\bar{z}^{*}\right)+s=\left\|z^{*}\right\|^{2}\right)$. Then, by the conditions

$$
\begin{aligned}
& f_{0}\left(\bar{z}^{0}\right)+s \leq\left\|\bar{z}^{0}\right\|^{2}, \\
& f_{0}\left(\bar{z}^{*}\right)+s \geq\left\|\bar{z}^{*}\right\|^{2}
\end{aligned}
$$

and $\left\|\bar{z}^{0}\right\|^{2} \leq\left\|\bar{z}^{*}\right\|^{2}$ implies $f_{0}\left(\bar{z}^{*}\right) \geq f_{0}\left(\bar{z}^{0}\right)$, whence, $f_{0}\left(\bar{z}^{*}\right)=f_{0}\left(\bar{z}^{0}\right)$. Similarly, by the conditions

$$
\begin{aligned}
& f_{0}\left(\bar{z}^{0}\right)+s=\left\|z^{0}\right\|^{2}, \\
& f_{0}\left(\bar{z}^{*}\right)+s=\left\|z^{*}\right\|^{2}
\end{aligned}
$$

and $\left\|z^{0}\right\|^{2} \leq\left\|z^{*}\right\|^{2}$ follows $f_{0}\left(\bar{z}^{*}\right) \geq f_{0}\left(\bar{z}^{0}\right)$, whence, again $f_{0}\left(\bar{z}^{*}\right)=f_{0}\left(\bar{z}^{0}\right)$. The theorem is proved.

Let $\left(z^{*}, d^{*}\right)$ be the solution of the problem (4). If $r\left\|z^{*}\right\|^{2}=d^{*}$ holds then $z^{*}$ is the solution to the problem (1). Otherwise, we will solve a problem (4) for the fixed value of a variable $d$. Let for $d=d^{*}$ the condition $r\left\|z^{*}\right\|^{2}=d^{*}$ be satisfied. By this value $d^{*}$ we will find a dichotomy method. If $d^{*}$ is minimum then $z^{*}-$ the solution to the problem (1).

Consider the problem

$$
\max \left\{\|z\|^{2} \mid g_{i}(z) \leq d, i=0, \ldots, m, z \geq 0\right\}
$$

where all $g_{i}(z), i=0, \ldots, m$ are strong convex functions.

For special cases, a convex feasible domain of the problem (6) is convex polyhedron or intersection of the balls. It is easy to prove the following theorem.

Let $S$ is feasible domain of the problem (6). The convex set can be described of a ball.

Theorem 2. Let $S$ be convex set, $S=\left\{x\|x-c\|^{2} \leq r^{2}\right\}$ and $x^{*}-$ the solution to the convex problem

$$
\max \left\{c^{T} x \mid x \in S\right\}
$$

then $x^{*}-$ the point of global maximum of problem 


$$
\max \left\{\|x\|^{2} \mid x \in S\right\}
$$

if $\left\|x^{*}-c\right\|^{2}=r^{2}$.

Proof. The proof follows from this, the side $\left(0, x^{*}\right)$ of the triangle $\left(0, x^{*}, c\right)$ is more than the side $\left(0, x^{0}\right)$ of the triangle $\left(0, x^{0}, c\right)$ for any $x^{0} \in S$.

Any convex set can be described of a convex polyhedron $P$. Then the problem (6) is approximated by the following

$$
\max \left\{\|x\|^{2} \mid A x \leq b, x \geq 0\right\} .
$$

We use exact quadratic regularization and have

$$
\begin{gathered}
\max \left\{\|x\|^{2} \mid a_{i}{ }^{T} x+\|x\|^{2}-b_{i} \leq d, i=1, \ldots, m,\right. \\
\left.\|x\|^{2}-x_{i} \leq d, i=1, \ldots, n\right\} .
\end{gathered}
$$

Feasible domain of this problem is intersection of balls.

\section{a. Feasible domain is intersection of balls}

Any convex feasible domain can be approximated by intersection of the balls. We show that the solution of the problem

$$
\max \left\{\|x\|^{2}\left\|x-a^{i}\right\|^{2} \leq r_{i}^{2}, i=1, \ldots, m\right\}
$$

can be found by a dual method. Let us notice that the problem (9) can have the duality gap that is nonzero.

The Lagrange function of the problem (9) can be written as

$$
L(x, \lambda)=\|x\|^{2}-\sum_{i=1}^{m} \lambda_{i}\left(\left\|x-a^{i}\right\|^{2}-r_{i}^{2}\right)
$$

The method of multipliers by Lagrange allows to find the solution to the problem (9)

$$
x=\frac{\sum_{i=1}^{m} \lambda_{i} a^{i}}{\sum_{i=1}^{m} \lambda_{i}-1}
$$

Thus, for solving problem (9) it is necessary to define optimal Lagrange multipliers $\lambda \geq 0$. If the point of global maximum $x^{*}$ is known, then we find Lagrange multipliers solving the system of linear equations

$$
x^{*}-\sum_{i=1}^{m} \lambda_{i}\left(x^{*}-a^{i}\right)=0
$$

For $m>n$ this system has many solutions, but only one solution coincides with optimal Lagrange multipliers of the problem (9). We write down Lagrange function (10) in a form

$$
L(x, \lambda)=\|x\|^{2}-\sum_{i=1}^{m} \lambda_{i}\left(\|x\|^{2}-2 x a^{i}+\left\|a^{i}\right\|^{2}-r_{i}^{2}\right)
$$

or

$$
L(x, \lambda)=\left(1-\sum_{i=1}^{m} \lambda_{i}\right)\|x\|^{2}+2 \sum_{i=1}^{m} \lambda_{i} x a^{i}+\sum_{i=1}^{m} \lambda_{i}\left(\mid a^{i} \|^{2}-r_{i}^{2}\right)
$$

that is transformed into

$$
\begin{aligned}
& L(x, \lambda)=\left(1-\sum_{i=1}^{m} \lambda_{i}\right)\left\|x+\frac{\sum_{i=1}^{m} \lambda_{i} a^{i}}{1-\sum_{i=1}^{m} \lambda_{i}}\right\|^{2}-\frac{\left\|\sum_{i=1}^{m} \lambda_{i} a^{i}\right\|^{2}}{\left(1-\sum_{i=1}^{m} \lambda_{i}\right)}- \\
& -\sum_{i=1}^{m} \lambda_{i}\left(\left\|a^{i}\right\|^{2}-r_{i}^{2}\right) .
\end{aligned}
$$

Substituting the solution (11) into formula (12) we obtain a dual problem

$\min \left\{\frac{\left\|\sum_{i=1}^{m} \lambda_{i} a^{i}\right\|^{2}}{\sum_{i=1}^{m} \lambda_{i}-1}-\sum_{i=1}^{m} \lambda_{i}\left(\left\|a^{i}\right\|^{2}-r_{i}^{2}\right) \mid \sum_{i=1}^{m} \lambda_{i}-1 \geq 0, \lambda \geq 0\right\}$.

Add the constraints of an initial problem into constraints of dual problem (13), expressed by dual variables. We obtain the dual convex problem

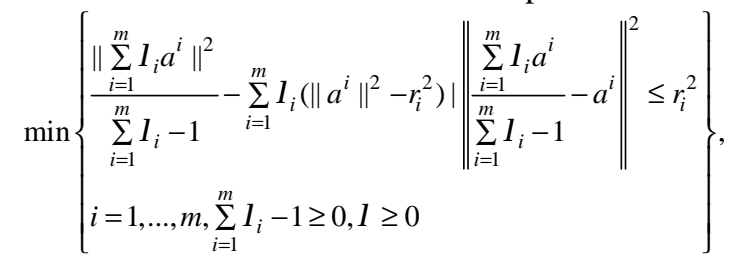

The objection function of dual problem is convex, when $p>0$, where

$$
p=\sum_{i=1}^{m} \lambda_{i}-1 .
$$

If the condition $p>0$ holds for the optimal Lagrange multipliers then they are the solution to the problem (9). We find the solution to the problem (9) by the formula (11). If $p \leq 0$ for the optimal Lagrange multipliers then the problem (14) has non-optimal Lagrange multipliers. These multipliers define the solution to the problem (9) $x^{*}$ by the formula (11). We substitute $x^{*}$ into the system

$$
x^{*}-\sum_{i \in I} \lambda_{i}\left(x^{*}-a^{*}\right)=0
$$

where $I$ is the set of active constraints in problem (9). Then, the solution of (15) is optimal Lagrange multipliers. We find optimal Lagrange multipliers in the following example

$$
\begin{aligned}
& \min \left\{\|x\|^{2} \mid\left(x_{1}+2\right)^{2}+\left(x_{2}-2\right)^{2} \leq 16,\left(x_{1}+2\right)^{2}+\right. \\
& +\left(x_{2}+2,5\right)^{2} \leq 30, \\
& \left.\left(x_{1}-0,5\right)^{2}+\left(x_{2}-0,5\right)^{2} \leq 6\right\} .
\end{aligned}
$$

For a dual problem (14) the solution is

$$
\lambda=(0.02230177,0.177738467,0.80006496) \text {. }
$$

It does not coincide with optimum multipliers. This solution defines the point $x^{*}=(0.456183345$, 2.755152574) of global maximum problem (9). Its optimal multipliers $\lambda^{*}=(0,0.1887523,0.78185117)$ are found by the following system

$$
\begin{aligned}
& -0.456183345=\lambda_{2}(-0.456183345+2)+ \\
& +\lambda_{3}(-0.456183345-0.5),
\end{aligned}
$$




$$
\begin{aligned}
& 2,755152574=\lambda_{2}(2.755152574+2.5)+ \\
& +\lambda_{3}(2.755152574-0.5) .
\end{aligned}
$$

If the problem (15) has no solution then we add the artificial constraint $\left\|x-a^{m+1}\right\|^{2} \leq r_{m+1}^{2}$ where $a^{m+1}$ and $r_{m+1}$ are chosen such as $\left\|x^{*}-a^{m+1}\right\|^{2}<r_{m+1}^{2} \quad$ and $x^{*}-a^{m+1}<0$. Adding a new constraint increases the $\lambda$ values in proportion to the value $x^{*}-a^{m+1}<0$.

Theorem 3. If the problem (9) has the solution then the solution $\lambda *$ to a modified dual problem

$$
\min \left\{g(\lambda) \mid 1 \leq \sum_{i=1}^{m} \lambda_{i},\left\|x(\lambda)-a^{i}\right\|^{2} \leq r_{i}^{2}, i=1, \ldots, m, \lambda \geq 0\right\}
$$

will define a point of a global maximum $x^{*}=x(\lambda *)$ of the problem (9).

Prof. Let us present constraints of a problem (9) in the form

$$
\|z(\lambda)\|^{2}-2 z(\lambda)^{T} a^{i}+\left\|a^{i}\right\|^{2} \leq r_{i}^{2}, i=1, \ldots, m .
$$

We will multiply both parts of inequalities on $\lambda_{i}$ and we will find their sum, receive

$$
\|z(\lambda)\|^{2} \sum_{i=1}^{m} \lambda_{i}-2 z(\lambda) \sum_{i=1}^{m} \lambda_{i}+\sum_{i=1}^{m} \lambda_{i}\left(\left\|a^{i}\right\|^{2}-r_{i}^{2}\right) \leq 0
$$

and use (4) can be expressed as

$$
\sum_{i=1}^{m} \lambda_{i}\left(\left\|a^{i}\right\|^{2}-r_{i}^{2}\right) \leq\left(\sum_{i=1}^{m} \lambda_{i}-2\right)\|z(\lambda)\|^{2} .
$$

Whence it follows that $\|z(\lambda)\|^{2} \leq g(\lambda)$ as

$$
g(\lambda)=\left(\sum_{i=1}^{m} \lambda_{i}-1\right)\|z(\lambda)\|^{2}-\sum_{i=1}^{m} \lambda_{i}\left(\left\|a^{i}\right\|^{2}-r_{i}^{2}\right) .
$$

Let the solution of dual problem $\lambda^{0}$ define only a point of a local maximum of a problem (9). Then to the global solution of a problem (9) there will correspond other point $\lambda^{*}$. Convex set $\left\{\lambda \mid g(\lambda) \leq g\left(\lambda^{*}\right)\right\}$ will contain interior points of feasible set of a problem (16), otherwise the point $\lambda^{*}$ will be also the solution to the problem (16). However, that contradicts convexity of a problem (16). These interior points will be interior and for set $\left\{\lambda \| z(\lambda) \leq z\left(\lambda^{*}\right)\right\}$ but then for these interior points inequality $\|z(\lambda)\|^{2}>g(\lambda)$ holds. Then we have a contradiction. It proves that the solution to the problem (16) defines a point of a global maximum of a problem (9).

We propose the following algorithm for the solution to the problem (9):

Step 1. We solve problem (14) and check duality gap. If it is equal to zero then the problem (9) is solved.

Step 2. We calculate the point $x^{*}$ by the formula (11) and define these of active constraints at this point. We solve a linear system of the equations (15) and define optimal Lagrange multipliers.

Step 3. For the found multipliers we check duality gap. If it is equal to zero the problem (9) is solved. Otherwise, we add new constraint in a problem (9) and pass to Step 1.

\section{GENERAL QUADRATIC OPTIMIZATION}

Consider the problem (1) where

$$
f_{i}(x)=x^{T} A_{i} x+b_{i}^{T} x+c_{i}, i=1, \ldots, m .
$$

Then the problem (1) will be transformed to the following

$$
\begin{aligned}
& \min \left\{u_{0}^{T} x^{2}+v_{0}^{T} x+w_{0} \mid u_{i}^{T} x^{2}+v_{i}^{T} x+w_{i} \leq 0,\right. \\
& i=1, \ldots, m, P x=q\}
\end{aligned}
$$

where $u^{T} x^{2}=u_{1} x_{1}^{2}+\ldots+u_{n} x_{n}^{2}$ or

$$
\begin{aligned}
& \min \left\{u_{0}^{T} y+v_{0}^{T} x+w_{0} \mid u_{i}^{T} y+v_{i}^{T} x+w_{i} \leq 0,\right. \\
& \left.i=1, \ldots, m, P x=q, x_{j}^{2}=y_{j}, j=1, \ldots, n\right\} .
\end{aligned}
$$

We use exact quadratic regularization and obtain

$$
\max \left\{\|z\|^{2}\right\}
$$

subject to

$$
\begin{aligned}
& u_{0}^{T} y+v_{0}^{T} x+w_{0}+s+\|z\|^{2} \leq d, \\
& \mid u_{i}^{T} y+v_{i}^{T} x+w_{i}+2\|z\|^{2} \leq d, i=1, \ldots, m, \\
& p_{j}^{T} x+2\|z\|^{2}-q \leq d, j=1, \ldots, k, \\
& x_{j}^{2}-y_{j}+2\|z\|^{2} \leq d, j=1, \ldots, n \\
& -\sum_{j=1}^{k}\left(p_{j}^{T} x-q_{j}\right)+2\|z\|^{2} \leq d, \\
& \sum_{j=1}^{n}\left(y_{j}-x_{j}^{2}\right)+2\|z\|^{2} \leq d .
\end{aligned}
$$

where $z=\left(x, x_{n+1}, y, q\right)$.

Table 1

The comparative numerical experiments

\begin{tabular}{|c|r|r|r|r|c|}
\hline Problem & $n$ & $m$ & $\begin{array}{c}\text { Method EQR } \\
\text { glob. min. }\end{array}$ & $\begin{array}{c}\text { The best } \\
\text { known } \\
\text { glob. min. }\end{array}$ & Ref. \\
\hline $\begin{array}{c}\text { Egg Holder } \\
\text { Rana }\end{array}$ & 100 & 0 & $\mathbf{- 8 9 9 4 8 . 5 3 2}$ & -89938 & {$[5]$} \\
\hline Nie & 50 & 0 & $\mathbf{- 5 0 8 5 5 . 7 8 4}$ & -41047.18 & {$[5]$} \\
\hline Nie & 49 & 1 & $\mathbf{- 9 . 9 8 2 8 4 6 2 9}$ & -0.5322069 & {$[6]$} \\
\hline Nie & 50 & 0 & $\mathbf{- 1 8 0}$ & -156 & {$[6]$} \\
\hline Nie & 40 & 0 & $\mathbf{- 1 5 6 0}$ & -1550.5 & {$[6]$} \\
\hline meanvar & 9 & 2 & 4,735427246 & 5,243399 & GL \\
\hline G16 & 5 & 38 & $\mathbf{1 . 9 1 4 6 0 8}$ & -1.9046617 & {$[7]$} \\
\hline Charles_Audet & 16 & 21 & $\mathbf{1 5 6 . 2 1 9 6 2 9 3}$ & 174.788 & {$[8]$} \\
\hline Ex7_3_5 & 13 & 15 & $\mathbf{0 . 0 2 4 9 9 6 7}$ & 1.206897 & GL \\
Ex8_4_7 & 63 & 41 & $\mathbf{2 6 . 9 9 4 3 0 9}$ & 28.898 & GL \\
\hline Ex6_2_5 & 10 & 3 & $\mathbf{- 7 0 . 9 5 8 6}$ & -70.75 & GL \\
\hline Ex2_1_8 & 24 & 10 & $\mathbf{1 5 6 3 9}$ & 15990 & GL \\
\hline Haverly & 12 & 9 & $\mathbf{- 4 0 6}$ & -400 & GL \\
\hline Harker & 20 & 7 & $\mathbf{- 1 0 2 0 . 2 4 2 9 8}$ & -986.513 & GL \\
\hline
\end{tabular}

Feasible domain of this problem is intersection of balls and ellipsoids. These ellipsoids are almost the balls. They coincide with all QCQP. We will replace ellipsoids by the balls.

The accuracy of the approximation satisfies the condition

$$
\Delta=\sqrt{\frac{d}{2}}\left(\frac{1}{\sqrt{1-\alpha / 2}}-1\right) .
$$




\section{NUMERICAL EXPERIMENTS}

In this section, we check the practical performance of method EQR. We have found solutions to more than 350 difficult test problems (see example:

http://www.gamsworld.org/global/globallib.htm_).

Some results are shown in Table 1 . These known test problems were solved by different methods during many years.

\section{CONCLUSION}

We have solved many difficult optimizing problems in optimal designing, clustering, sensor networks and chemistry [Kos]. The EQR method can be used for the solution of discrete problems. We offer for the first time a method for solving classes of multiextreme problems.

The comparative numerical experiments have shown that new methods are very efficient and promising.

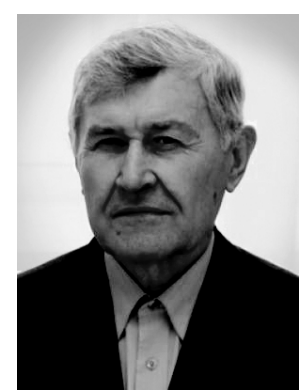

Anatolii Kosolap is the doctor of physical and mathematical sciences, the professor, Head of the Department of specialised computer systems of the University of Chemical Engineering.

Area of scientific interests: mathematical modelling, the con-

\section{REFERENCES}

[1] A. Kosolap, Methods of Global Optimization. Dnipropetrovsk: Science and education, 2013 (in russian).

[2] V. P. Kenneth, R. M. Storn, and J. A. Lampinen, Differential Evolution. A Practical Approach to Global Optimization. Berlin, Heidelberg: Springer-Verlag, 2005.

[3] J. Nocedal, and S.J. Wright, Numerical optimization. Springer, 2006.

[4] Y. Ye, Semidefinite programming. Stanford University, 2003.

[5] A. P. Piotrowski and J. J. Napiórkowski, (2010) The grouping dierential evolution algorithm for multi-dimensional optimization problems. Control and Cybernetics, vol. 39, No. 2, pp. 527-550.

[6] J. Nie, Regularization Methods for Sum of Squares Relaxations in Large Scale Polynomial Optimization. University of California, 2009.

[7] M. K. Dhadwal, S. N. Jung, C. J. Kim, (2014) Advanced particle swarm assisted genetic algorithm for constrained optimization problems. Comput. Optim. Appl., 58, pp. 781-806.

[8] C. Audet, P. Hansen, B. Jaumard, G. Savard, (2000) A branch and cut algorithm for nonconvex quadratically constrained quadratic programming. Math. Program., Ser. A 87, pp. 131-152.

vex analysis, the theory and numerical methods of finite dimensional optimization.

He has offered new effective methods for the solution problems of the global, semidefinite, nonsmooth, combinatory optimization. He has developed the polyconvex analysis, generalised the theory of a duality for multimodal problems.

The author of six monographies and more than 200 works in optimization. 